\title{
Prenatal Diagnosis of Teratoma in a Torqued Undescended Testis Masked as Unclear Intra-Abdominal Mass
}

\section{Introduction}

Constantly improving ultrasound technologies facilitate prenatal detection of various fetal diseases, including low-incidence disorders that are typically diagnosed postnatally, such as testicular teratoma. These can appear as an unclear cystic intra-abdominal structure, caused by an undescended torqued testis. In the case of such uncertain cystic intra-abdominal lesions, a cryptorchidism could lead the investigator to narrow the possibilities to the urogenital tract.

Herein, we report the second case of a prenatally detected cystic-solid lesion being a testicular teratoma by a torqued undescended testis and the seventh case of a prenatally seen testicular teratoma. In late gestation, a structured evaluation of the testes would be beneficial to detect such malformations.

\section{Case Report}

A 24-year-old woman, gravida 3 para 0 , was referred to our department at $30+3$ weeks with an undefined cystic intra-abdominal mass in a male fetus. Ultrasound examination confirmed the predominantly cystic tumor with a small solid central part $(8 \times$ $13 \mathrm{~mm}$ ), measuring a total size of $30.4 \times$ $22.9 \times 30.2 \mathrm{~mm}$, without vascular flow. The tumor was located between the right lower renal pole and the bladder ( $\triangleright$ Fig. $\mathbf{1})$. In its largest sagittal extension, the tumor reached into the right middle abdomen and was in cranial contact with the lower liver edge. Based on clinical features, a peritoneal cyst or mesenteric cyst was suspected. Further sonographic examinations at $34+4$ and $40+0$ weeks showed no signs of progress. At $40+2$ weeks, a 3660-g male fetus with Apgar scores of 9, 10, and 10 at 1, 5 and $10 \mathrm{~min}$, respectively, was delivered vaginally.

Postnatal ultrasound examination confirmed the prenatal findings. Moreover the heterogeneous, well-defined round mass $(19 \times 26 \times 23 \mathrm{~mm})$ showed central calcifications, as well as intralesional septations with an absence of vascular flow. There was no evidence of affected lymph nodes, as- cites, intestinal or urinary tract obstructions. An abdominal teratoma was assumed and laparotomy was performed on day 8 of life.

A $28 \times 22 \times 16 \mathrm{~mm}$ beige-colored hard tumor, which most closely resembled a torqued testis, was completely resected. Histological diagnosis was teratoma in a necrotic right testis with focal inclusion of mature cell bone and cartilage tissue without evidence of malignancy. Necrotic changes were attributed to prenatal torsion.

The infant recovered well and was dismissed on the third day after surgery with normal serum AFP (preoperative $7311 \mathrm{ng} /$ $\mathrm{ml} v \mathrm{~s} .1660 \mathrm{ng} / \mathrm{ml}$ on the third postoperative day) levels.

\section{Discussion}

Most prenatally detected fetal intra-abdominal cystic lesions in the second or third trimester, including multicystic dysplasia, other malformations of the urinary tract, and hydronephrosis resembling such lesions, originate from the kidney. Abnormalities of the adrenal glands, such as neuroblastoma, and adrenal hemorrhage, cystic lesions of the liver and biliary tract, spleen, lymphatic system, peritoneum, omentum, and retroperitoneal or sacrococcygeal tumors, may also appear the same way (GE.
Hartmann et al. Clin Perinatol 1989; 16: 123-135).

Prepubertal teratoma in an undescended torqued testis is an extremely rare phenomenon and is therefore often missed when considering possible fetal prenatal differential diagnoses upon observing such an intra-abdominal lesion (GM. Janda et al. Urology 2014; 83:214-216). The incidence of prepubertal testicular tumors is 0.5 in 100000 in general, whereas no incidence rate is given directly for testicular teratomas. Depending on the given data collection, testicular teratoma is accountable for $23-48 \%$ of all prepubertal testicular tumors. Furthermore, a differentiation between descended and undescended testis would be necessary in those cases for determining an unambiguous incidence rate.

At present, testicular teratoma is usually diagnosed at the age of 13 months, typically as a swelling mass. Introduction of a structured prenatal examination of the testis and the findings of a cryptorchidism could guide the examiner to the affected organ and indicate the rare diagnosis. The embryological chronological sequence of the descent of the testis could therefore be used as a guideline for sonographic examination. Knowledge of the testes typically reaching the inguinal region by 12 weeks of gestation, migrating through it by 28 weeks

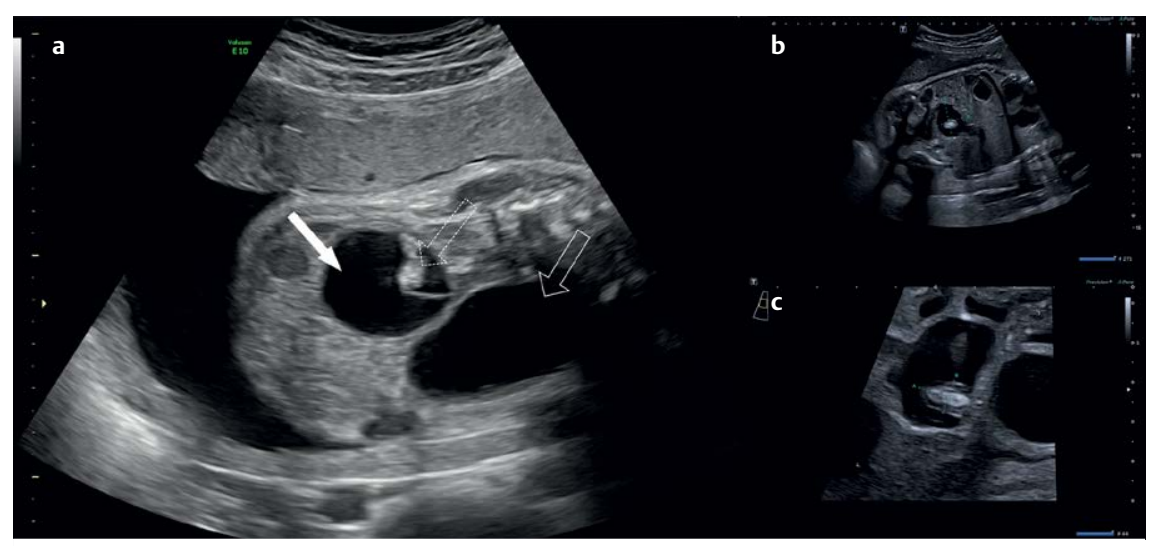

- Fig. 1 Prenatal ultrasound images demonstrating teratoma in a torqued undescended testis at $30+3(1 \mathbf{a})$ and $34+5$ weeks of gestation ( $1 \mathbf{b}$ and $1 \mathbf{c}$, respectively). The filled arrow indicates the cystic part and the dotted arrow the solid part of the torqued testis. The continuous arrow points to the fetal urinary bladder (1a). The intraabdominal tumor measures a total size of 28.9 $\times 21.7 \mathrm{~mm}$, and the solid part measures a total size of $13.2 \times 8.5 \mathrm{~mm}(1 \mathbf{b}$ and $\mathbf{c})$. 
and finally getting to the scrotum by 33 weeks could provide orientation for the sonographic examination (R. Achiron et al. Ultrasound Obstet Gynecol 1998; 11:242245). Vascularity should also be assessed in this context, as bleeding from teratomas is not uncommon and can lead to fetal anemia.

As seen in our case, after the abdominal cystic lesion was diagnosed, a structured prenatal ultrasound examination of the testis was not performed and the possibility of prepubertal teratoma of the testis was not considered.

In addition, a structured prenatal ultrasound examination of the testis could influence the perinatal management and therefore alter the neonatal outcome. Knowledge of testicular descent times during prenatal life may be helpful when considering the differential diagnosis of abdominal cystic lesions in male fetuses. If diagnosis of a testicular teratoma is considered, early referral to a pediatric surgeon could be made and complications such as torsion could be prevented. If possible, a testis-sparing operation could be performed. Birth should take place in a tertiary referral center and parents must be informed about necessary neonatal follow-up and risk factors as a result of the diagnosis. Further investigations of this rare phenomenon are necessary.

\section{Conflict of Interest}

The authors declare that they have no conflict of interest.

Authors

Adeline Walter $^{1}$ (D), Alexander C. Engels ${ }^{1}$, Andreas Heydweiller ${ }^{2}$, Annegret Geipel', Ulrich Gembruch ${ }^{1}$

\section{Affiliations}

1 Department of Obstetrics and Prenatal Medicine, University Hospital Bonn, Bonn, Germany

2 Department of Pediatric Surgery, University Hospital Bonn, Bonn, Germany
Correspondence:

Mrs. Adeline Walter

Department of Obstetrics and Prenatal Medicine, University Hospital Bonn Venusberg-Campus 1

53127 Bonn

Germany

Tel .: + 49 (0)228-287-15449, Fax : + 49

(0)228-287 16088

adeline.k@live.de

Bibliography

DOI https://doi.org/10.1055/a-1088-3569 Ultrasound Int Open 2019; 5: E96-E97 (c) Georg Thieme Verlag KG Stuttgart · New York

ISSN 2199-7152

\section{() (1) $\Theta \Theta$}

